\title{
First report of the macrolide efflux genetic assembly (MEGA) element in Haemophilus parainfluenzae
}

Sir,

We previously described two identical extensively drug-resistant (XDR) Haemophilus parainfluenzae isolates non-susceptible to $\beta$-lactams, macrolides, quinolones, tetracycline and chloramphenicol [1]. In an effort to analyse how the resistance genes of these pathogens were organised, one isolate (AE-2096513) underwent Illumina HiSeq 200 whole-genome sequencing. Genomic DNA was extracted and multiplexed pairedend libraries were created using the sample preparation kit from Illumina Inc. (San Diego, CA). SPAdes v.2.5 (kmer sizes $=33,55,67,81,91,93,95,97,99)$ was used for read correction and de novo assembly [2]. The resulting number of scaffolds and total sequence length was $2.71 \mathrm{Mb}$ (GenBank accession no. MOTP00000000). Scaffolds with a length of $<500 \mathrm{bp}$ were extracted, resulting in 124 scaffolds that had more than 100 -fold read coverage.

Special emphasis was then placed on the genetic environment of $m e f(E) / m e l$ (encoding a dual efflux pump affecting macrolides), often found within the macrolide efflux genetic assembly (MEGA) element, and tet(M) (encoding a ribosomal protective protein against tetracyclines). The Illumina reads were mapped using Bowtie2 v.2.1.0 to the MEGA element (accession no. FR671415). The reads pairs, of which only one mate mapped to the MEGA element, were extracted. The non-mapping mates were aligned to the 
genome sequence of the reference H. parainfluenzae T3T1 (accession no.

YP 004823607) using Basic Local Alignment Search Tool (BLAST) to specifically focus on the flanking regions of the tet(M)-MEGA element (accession no. $\underline{\text { KJ545575; see }}$ results below).

Using this information, a fusion sequence including the tet(M)-MEGA element was constructed in silico (Fig. 1). Based on this result, sequence primers were designed to confirm the chromosomal insertion site of the element using traditional Sanger sequencing. First, using the tet(M)-MEGA flanking primers RF-end (5'GGTTTAACCGAATGGGCGCCTGC-3') and 19210rev (5'CTTGCAGTCAAATAGTAGTTGG-3'), a PCR product with the expected insert site (ca. $10 \mathrm{~kb})$ was obtained. Second, it was revealed that the tet(M)-MEGA element was inserted 2 bp downstream of RF-3 (ORF19190) at position 1989391 compared with $H$. parainfluenzae T3T1 using primers RF-end and MEGA-end (5'TTTATTTAAGAATACCTTGCCGC-3'). Third, primers mef(E)F (5'TTCTTCTGGTACTAAAAGTGG-3') and 19210rev were used to obtain a PCR product of $2.1 \mathrm{~kb}$, which was subsequently sequenced using an additional internal primer (IMLS2, 5'-TCCCGCACCATTTATCAGCTT-3'). This revealed that the downstream insertion site of tet(M)-MEGA in AE-2096513 is at position 1989935 compared with T3T1, therefore replacing ORF19200 (Fig. 1). Finally, we closely inspected the ends of the element, showing that there are no inverted repeats rendering difficult a possible explanation for the mechanism of insertion (Fig. 1). 
The MEGA element has been previously reported in streptococcal species such as Streptococcus salivarius and Streptococcus pneumoniae [3]. For S. salivarius, no adjacent tet(M) was described, whilst for $S$. pneumoniae tet(M) upstream of the MEGA element and within transposon Tn916 has been frequently identified (Fig. 1). Tn916 and the often co-identified composite transposons (e.g. Tn2009 and Tn2010) are thought of being mobile by transformation, and more than four distinct insertion sites have been identified within the pneumococcal genome [4]. In AE-2096513, tet(M) together with MEGA were in a 'non-Tn916" context because Tn916 typically contains the ORF8 to 23 (as illustrated for S. pneumoniae 23771) that were absent in our element (Fig. 1).

In conclusion, we identified for the first time the tet(M)-MEGA element in an $H$. parainfluenzae isolate. This element is probably mobilised and might be transferred between different members of the microbial community. In the case of $H$. parainfluenzae AE-2096513, this strain has been isolated along with a pan-susceptible Neisseria gonorrhoeae from a urethral swab [1]. Haemophilus parainfluenzae was therefore considered as a coloniser, but it would be particularly worrisome if the newly described tet(M)-MEGA could be exchanged with $N$. gonorrhoeae, conferring resistance to azithromycin, one of the standard therapeutic options [5]. However, H. parainfluenzae is normally isolated from the pharynx, where there are also streptococci and pneumococci. Considering the close similarity of the tet(M)-MEGA elements of $H$. parainfluenzae AE2096513 and S. pneumoniae 23711, genetic exchange between these two bacterial species is likely. These findings may therefore serve as a paradigmatic example of 
microbial acquisition of mobile genetic elements carrying resistance genes against clinically used antibiotics.

Acknowledgments: The authors thank Regula Tinguely and Lea Hirzberger for their technical help.

Funding: This work was partly supported by the Swiss National Science Foundation [SNF No. 320030_159791].

Ethical approval: Not required.

\section{References}

[1] Tinguely R, Seiffert SN, Furrer H, Perreten V, Droz S, Endimiani A. Emergence of extensively drug-resistant Haemophilus parainfluenzae in Switzerland. Antimicrob Agents Chemother 2013;57:2867-9.

[2] Bankevich A, Nurk S, Antipov D, Gurevich AA, Dvorkin M, Kulikov AS, et al. SPAdes: a new genome assembly algorithm and its applications to single-cell sequencing. J Comput Biol 2012;19:455-77. 
[3] Santagati M, Lupo A, Scillato M, Di Martino A, Stefani S. Conjugal mobilization of the MEGA element carrying mef(E) from Streptococcus salivarius to Streptococcus pneumoniae. FEMS Microbiol Lett 2009;290:79-84.

[4] Gay K, Stephens DS. Structure and dissemination of a chromosomal insertion element encoding macrolide efflux in Streptococcus pneumoniae. J Infect Dis 2001;184:56-65.

[5] Unemo M, Shafer WM. Antimicrobial resistance in Neisseria gonorrhoeae in the 21st century: past, evolution, and future. Clin Microbiol Rev 2014;27:587-613.

Andrea Endimiani ${ }^{\text {a }}$

Aurélie Allemann ${ }^{\mathrm{a}, \mathrm{b}}$ Daniel Wüthrich ${ }^{\mathrm{c}}$ Agnese Lupo ${ }^{a, d}$ Markus Hilty ${ }^{\mathrm{a}, \mathrm{e}, *}$

a Institute for Infectious Diseases, Faculty of Medicine, University of Bern, Bern, Switzerland

${ }^{\mathrm{b}}$ Graduate School for Cellular and Biomedical Sciences, University of Bern, Bern,

Switzerland

${ }^{c}$ Interfaculty Bioinformatics Unit and Swiss Institute of Bioinformatics, University of

Bern, Bern, Switzerland

${ }^{d}$ Agence nationale de sécurité sanitaire de l'alimentation, de l'environnement et du travail (ANSES), Unité antibiorésistance et virulence bactériennes, Lyon, France 
e Department of Infectious Diseases, Bern University Hospital, University of Bern, Switzerland

* Corresponding author. Present address: Institute for Infectious Diseases, University of Bern, Friedbühlstrasse 51, Postfach 61, CH-3010 Bern, Switzerland. Tel.: +4131632

fax: +4131632 8766

E-mail address: Markus.Hilty@ifik.unibe.ch (M. Hilty) 
Fig. 1. Insertion of the tet(M)-MEGA element in the clinical Haemophilus parainfluenzae isolate (AE-2096513) found in Bern (Switzerland) and comparison with the elements previously reported in Streptococcus salivarius (AJ318993) and Streptococcus pneumoniae (FR671415). The tet(M) gene and open reading frames (ORFs) within MEGA are filled in black and grey, respectively. Labelling of ORF19190 and ORF19210 was done according to the deposited genome of $H$. parainfluenzae T3T1 (GenBank accession no. $\underline{\text { YP } \mathbf{0 0 4 8 2 3 6 0 7}})$. For T3T1, the tet(M)-MEGA element is indicated as a grey box and replaces ORF19200 (light grey box). The s/p gene encodes a surface lipoprotein. MEGA, macrolide efflux genetic assembly. 
H. parainfluenzae 